\title{
Rabaska
}

Revue d'ethnologie de l'Amérique française

\section{Du folklore à l'ethnologie : « traditions populaires " et projet universitaire en Bretagne. La contribution fondamentale de Jean-Michel Guilcher}

\section{Fañch Postic et Jean-François Simon}

Volume 13, 2015

Présence de Marius Barbeau : l'invention du terrain en Amérique française. Autour d’un legs centenaire (1914-2014)

URI : https://id.erudit.org/iderudit/1033766ar

DOI : https://doi.org/10.7202/1033766ar

Aller au sommaire du numéro

Éditeur(s)

Société québécoise d'ethnologie

ISSN

1703-7433 (imprimé)

1916-7350 (numérique)

Découvrir la revue

Citer cet article

Postic, F. \& Simon, J.-F. (2015). Du folklore à l'ethnologie : « traditions

populaires » et projet universitaire en Bretagne. La contribution fondamentale de Jean-Michel Guilcher. Rabaska, 13, 249-262.

https://doi.org/10.7202/1033766ar 


\title{
Du folklore à l'ethnologie : « traditions populaires » et projet universitaire en Bretagne. La contribution fondamentale de Jean-Michel Guilcher
}

\author{
Fañch Postic et Jean-François Simon \\ Université de Bretagne occidentale, Brest
}

\begin{abstract}
À considérer la localisation des structures dispensant aujourd'hui en France un enseignement complet d'ethnologie, allant de la licence au doctorat, il apparaît immédiatement que, sans les possibilités offertes par l'Université de Bretagne occidentale, le Grand-Ouest français aurait été de ce point de vue un espace désertique (ill. 1). Cette singularité brestoise, nous la devons pour une grande part à Jean-Michel Guilcher. Notre propos sera de rappeler comment une telle situation fut rendue possible, nous efforçant de replacer ce chercheur de portée internationale dans la position charnière qui fut la sienne, entre intérêts militants et exigences de la rigueur scientifique, entre méthodes et théories désuètes d'un folklore dépassé et principes et exigences d'une ethnologie du proche émergente. Cette évocation du parcours de Jean-Michel Guilcher, en parallèle à celui de Marius Barbeau, contribuera, nous l'espérons, à mieux appréhender les divergences entre les universités franco-canadiennes et françaises dans la prise en considération des « traditions populaires ».
\end{abstract}

\section{Avènement de l'ethnologie de la France}

C'est généralement à la création en 1937 à Paris, aux côtés du musée de l'Homme, du Musée national des arts et traditions populaires que ceux qui ont analysé la discipline ${ }^{1}$ situent l'avènement de l'ethnologie de la France.

1. Isac Chiva, « George[s-]Henri Rivière : un demi-siècle d'ethnologie de la France », Terrain, 5 octobre 1985, p. 76-83 ; id., «Émergence d'une ethnologie de la France. Essai de reconstitution généalogique ", Miroirs de l'Europe. La France et les pays de langue allemande [I. Chiva et U. Jeggle dir.], Paris, Msh, 1987, p. 9-33 ; Martine Segalen et Jean Cuisenier, Ethnologie de la France, Paris, PuF, « Que sais-je ? » n² 2307,1986; Martine Segalen, Vie d'un musée 1937-2005, Paris, Stock, 2005 ; Daniel Fabre, " Le Manuel de folklore français d'Arnold Van Gennep », Les Lieux de mémoire (sous la direction de Pierre Nora), vol. III, Les France, t. 2, Traditions, p. 641-675; Du folklore à l'ethnologie [J. Christophe, D.-M. Boëll, R. Meyrand [dir.], Paris, Éditions de la maison des sciences de l'homme, 2009. 


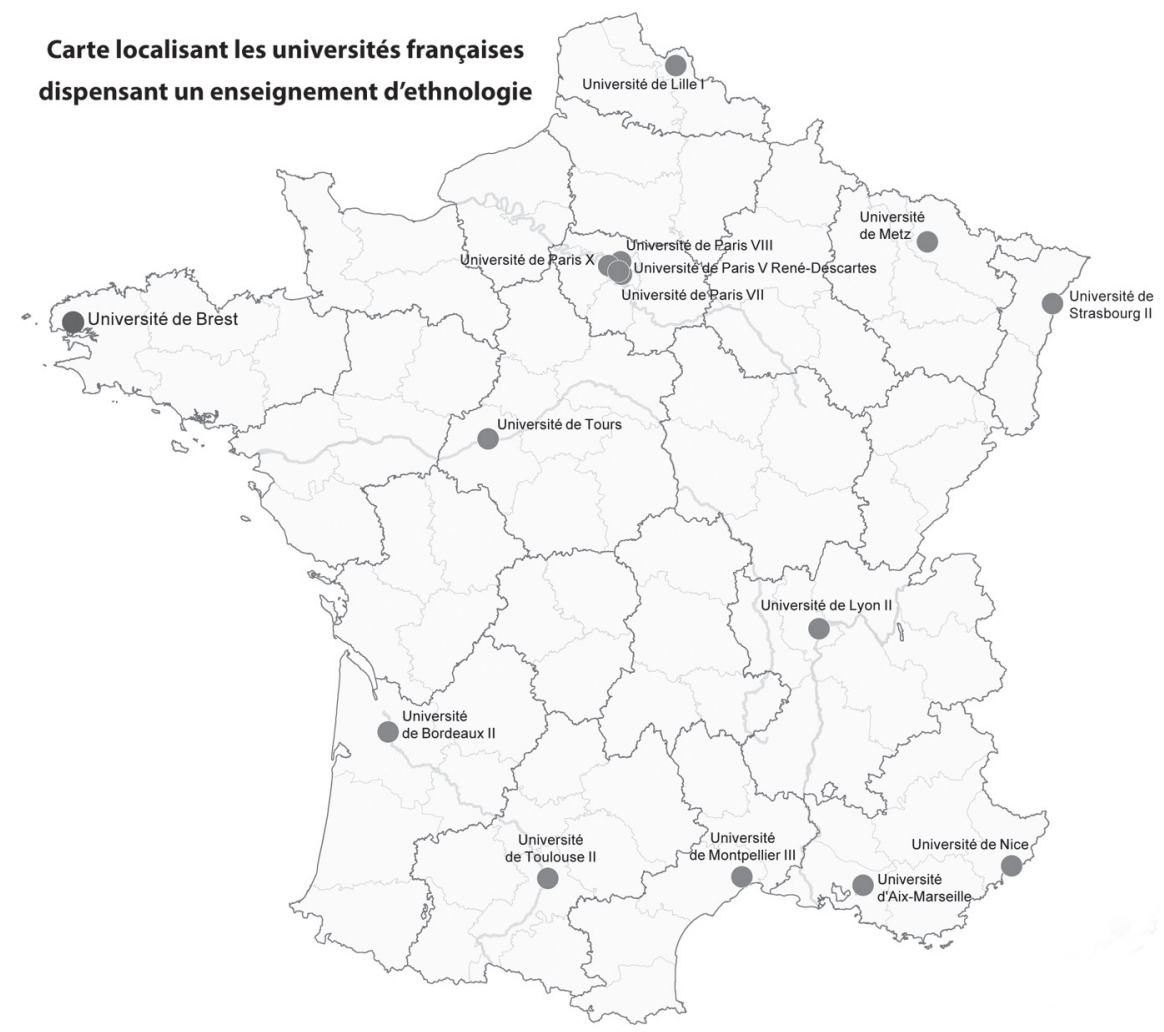

Illustration 1

Les enseignements d'ethnologie en France

Carte dessinée par Céline Siguret, Uво, Brest

D'abord « ethnographie française », cette dernière a bien du mal à se faire une place aux côtés de l'ethnologie de l'exotisme, et à se débarrasser d'origines qui prennent naissance dans le folklore, un terme qui, en France, a vite pris une connotation péjorative d'amateurisme et de passéisme, renforcée par l'utilisation qu'en fera le régime de Vichy.

Ceux qui s'intéressent alors à un champ de recherche qui, il convient de le souligner, n'a encore aucune visibilité institutionnelle, ne pouvaient évidemment se faire une place au sein de ladite institution. C'est le cas des Arnold Van Gennep (1873-1957), l'inventeur des rites de passage, de Patrice Coirault (1875-1959) et Paul Delarue (1889-1956), respectivement spécialistes de la chanson folklorique et du conte populaire. Ils se comportent, par nécessité, sinon en « francs-tireurs », du moins en électrons libres : ils s'investissent personnellement dans leurs travaux, y compris financièrement, et sont des indépendants, parfois des solitaires : certains, à l'image de Patrice Coirault 
se retirent dans l'intimité de leur cabinet de travail ; d'autres parviennent à trouver « refuge » auprès du Musée des arts et traditions populaires, à l'image de Delarue ou de Van Gennep, même si les relations de ce dernier, « anarchiste de conviction », avec Georges-Henri Rivière, le conservateur du musée, sont loin d'être simples².

Cela tient aux efforts constants, voire aux habiles manœuvres de Rivière, particulièrement soucieux d'engager des recherches autour du nouveau musée. Il s'attache notamment à établir des liens privilégiés avec le Centre national de la recherche scientifique, créé en 1939 et réorganisé au lendemain de la Seconde Guerre mondiale. Rivière est membre de la commission d'ethnographie du CNRS et, à ce titre, bien placé pour obtenir des moyens pour des missions ou des allocations pour des projets de recherche ${ }^{3}$. Ainsi en 1945, Van Gennep bénéficie-t-il d'une première aide (modeste) du CNRS qui lui permet de « consacrer toutes ses forces à la rédaction de son Manuel de folklore français contemporain..$^{4} »$ Mais Van Gennep a alors 72 ans ; Coirault en a 70 et Delarue près de 60.

Ce n'est effectivement que dans « la seconde moitié de la décennie 1950 » que, selon Martine Segalen, «s'affermit la politique de recherche du musée » des ATP, ouvrant la voie à « un pôle scientifique majeur et unique, à l'échelle de la France, [ « c'est le musée-laboratoire » cher à Rivière] mais cela plus de dix années après le redémarrage de l'ethnologie hors métropole..$^{5}$ »

C'est dans cet environnement que s'inscrit Jean-Michel Guilcher, dont le parcours scientifique nous semble présenter deux versants.

\section{Le versant personnel : entre promotion de l'éducation nouvelle et recherche personnelle ${ }^{6}$}

\section{A. L'investissement dans l'éducation nouvelle}

Au sortir du lycée de Brest, ville où il est né en 1914 et où sa mère est institutrice, Jean-Michel Guilcher suit des cours à la Sorbonne avec l'objectif de devenir enseignant d'histoire naturelle ${ }^{7}$. Il y fait la connaissance d'Hélène,

2. Daniel Fabre, op. cit, p. 654. Ses amis 1'appellent familièrement « l'ermite de Bourg-laReine » (Cf. Georges-Henri Rivière, « Préface », Bibliographie des æuvres d'Arnold Van Gennep, Paris, Picard, 1964, p. [1].

3. Martine Segalen, Vie d'un musée 1937-2005, op.cit., p. 171.

4. Nicole Belmont, Arnold Van Gennep le créateur de l'ethnographie française, Paris, Petite bibliothèque Payot n²32, p. 9-10.

5. M. Segalen, op. cit., p. 171.

6. Les informations concernant la carrière de Jean-Michel Guilcher proviennent, pour une part, du long entretien (près de 5 heures) qu'il a accordé le 3 avril 2014 à Jean-Luc Germain pour le compte de la revue Bretagne Magazine Histoire. L'enregistrement, déposé dans les fonds sonores du CRBC, est accessible en ligne sur le site Cocoon (COllections de COrpus Oraux Numériques) du CNRs : purl.org/ poi/crdo.vjf.cnrs.fr/crdo-JAP_0001_SOUND. Nous signalerons les citations extraites de ce document par la mention « entretien JLG/BMH ».

7. Il se montre particulièrement intéressé par la zoologie, la botanique, la stratigraphie ou la 
sa future femme, qui, en 1937, l'entraîne dans les cours de danses de Miss Pledge qui, adepte de pédagogies nouvelles, a largement contribué à moderniser l'éducation physique en France, mais dont l'avant-gardisme n'a été reconnu que tardivement, après sa mort ${ }^{8}$. Anglaise arrivée en France en 1926, elle enseigne la gymnastique, mais aussi le jeu et la danse populaire ${ }^{9}$. Pour Hélène et Jean-Michel Guilcher, la danse est un simple plaisir qu'à aucun moment ils n'imaginent devenir une occupation professionnelle. Cela s'inscrit dans tout un mouvement de développement de l'éducation populaire, favorisé par l'institution des congés payés en 1936 par le Front populaire. Jean-Michel Guilcher prépare l'agrégation quand survient la guerre qui le voit, en 1940, démobilisé en zone sud. Là, il trouve à s'employer au mouvement de jeunesse Jeune France à Lyon, dont le penseur est le philosophe Emmanuel Mounier (1905-1950) qui dirige également la revue Esprit: ce dernier, sachant l'intérêt que Jean-Michel Guilcher porte aux danses traditionnelles, lui demande un article évoquant le plaisir que les jeunes prennent à les pratiquer, mais traitant aussi de leurs origines qu'à l'époque, dans une belle unanimité, on fait remonter aux Gaulois, voire à la préhistoire. Dès janvier 1941, Jean-Michel Guilcher rédige un essai interprétatif ${ }^{10}$ qu'il récuse totalement aujourd'hui ${ }^{11}$ et dont la "rêverie poétique " et " les interprétations puériles " seront sévèrement critiquées par Henri Davenson ${ }^{12}$. Ce dernier propose un point de vue très différent, reposant sur la théorie d'échanges constants sur une très longue durée entre élites et milieux populaires. Ces divergences radicales d'approches entraînent chez Jean-Michel Guilcher une interrogation sur les mécanismes d'apprentissage et de transmission des danses traditionnelles dont on n'avait plus la moindre idée dans les « sociétés citadines modernes ». Mais

paléontologie (entretien JLG/BMH). Dès 1937, il publie dans la Revue générale de botanique un article de physiologie végétale sur "L'Intensité respiratoire comparée de l'androcée et du gynécée » (t. 49, $\mathrm{n}^{\circ}$ 577, janvier 1937, p. 235-242). Il est également en 1957 l'auteur d'un ouvrage sur les fougères (Atelier du père Castor).

8. Jean Saint-Martin, « Les Origines oubliées de la gymnastique volontaire entre les deux guerres mondiales », Sport et genre - volume 3 Apprentissage du genre et institutions éducatives [Jean SaintMartin et Thierry Terret dir.], Paris, L'Harmattan, 2005, p. 55-69.

9. Voir Alick-Maud Pledge (1893-1949). Voir l'article que lui a consacré Yves Guilcher : hmenf. free.fr/article.php3?id_article $=225$.

10. Jean-Michel Guilcher et Jean-Marie Serreau, « Le Chant et la danse populaire. Éléments de culture française », Esprit, janvier 1941. S'il porte une double signature, Jean-Michel Guilcher dit en assumer seul la paternité et « les insanités » qu'il contient. Il affirme ne l'avoir jamais relu depuis ! (entretien JLG/BMH)

11. Jean-Michel Guilcher, Danse traditionnelle et anciens milieux ruraux français. Tradition, Histoire, Société, Paris, L'Harmattan, 2009, p. 235, note 4.

12. La réponse se trouve dans le n ${ }^{\circ} 98$ de la revue Esprit de mars 1941, p. 358-359. Henri Davenson est le pseudonyme d'Henri-Irénée Marrou (1904-1977); historien, il s'intéresse également à la musique et on lui doit notamment Le Livre des chansons ou Introduction à la connaissance de la chanson, Paris, Le Seuil, 1944, où il rappelle (p. 34) "l'amicale polémique » qui l'a opposé à Serreau et Guilcher. Marrou sera le président du jury de thèse de Jean-Michel Guilcher sur la danse en Basse-Bretagne. 
comment en savoir plus sur un champ que personne n'avait pris en considération jusqu'alors, que les folkloristes avaient pour ainsi dire totalement ignoré ? En août 1941, l'association Jeune France le charge d'une mission pour recueillir le répertoire de danses de vallées pyrénéennes où il avait déjà réalisé une petite collecte un an plus tôt ${ }^{13}$. Jean-Michel Guilcher en vient à s'interroger sur l'existence d'autres milieux ruraux où il serait encore possible d'observer la manière dont les danses ont été apprises, vécues et transmises et cela sur plusieurs générations. Dans l'esprit de Jean-Michel Guilcher, formé aux exigences scientifiques de l'histoire naturelle qui veut que les hypothèses soient vérifiées par l'expérimentation, germe l'idée, nouvelle pour l'époque, de commencer par une observation directe sur le terrain, qui irait bien au-delà de la simple collecte d'un répertoire et qu'il imagine, comme une évidence, être en Bretagne, dans la région dont sa famille maternelle est originaire : la côte en face de l'île d'Ouessant. Mais l'Occupation ne le permet pas d'autant que la commune littorale de Porspoder est située en zone interdite.

En 1942, Jeune France est dissoute et Jean-Michel Guilcher entre dans la maison d'édition du Père Castor fondée en 1931 par Paul Faucher (1898-1967) ${ }^{14}$, promoteur de pédagogies nouvelles en matière d'éducation. Les Albums du père Castor sont déjà célèbres quand commence entre les deux hommes une étroite coopération que nourrit une réflexion sur les outils pédagogiques du futur : cela aboutit en 1945 à la création des « Ateliers du père Castor » auxquels Jean-Michel Guilcher collaborera activement pendant dix années.

\section{B. Une enquête personnelle sur les danses traditionnelles}

Parallèlement, à la Libération, Hélène et Jean-Michel Guilcher peuvent enfin engager en 1945, une enquête sur la côte nord du Finistère, « Le résultat de ce premier travail a été, précise Jean-Michel Guilcher, de nous apprendre à travailler. ${ }^{15}$ » C'est par simple esprit de curiosité qu'ils vont de proche en proche sans savoir ce qui allait en résulter réellement (ill. 2). C'est aussi en 1945 qu'ils rencontrent Patrice Coirault dont l'influence sera déterminante. «Dès l'été 1942, William Lemit m'avait fait lire Notre chanson folklorique. En 1945, il m'a présenté à M. et $\mathrm{M}^{\text {me }}$ Coirault, avec qui je n'ai plus cessé d'avoir des relations suivies. ${ }^{16} \gg$ Pendant dix ans, la recherche qu'ils mènent

13. Cette première enquête sera le sujet d'une conférence de Jean-Michel Guilcher à la Société d'ethnographie française le 11 décembre 1954 (Cf. Arts et Traditions populaires, avril-juin 1955, p. 152-153), puis d'une publication : " Deux danses du Lavedan », Arts et Traditions populaires, janv.-mars 1956, p. 1-29.

14. Le Père Castor Paul Faucher (1898-1967). Un Nivernais inventeur de l'album moderne, Actes du colloque de Pougues-les-Eaux, 20 et 21 novembre 1998, suivis des témoignages des collaborateurs de Paul Faucher, Conseil général de la Nièvre, 1999, 174 p.

15. Entretien JLG/BMH.

16. Yves Defrance et Jean-Michel Guilcher, « Jean-Michel Guilcher. Un demi-siècle de 


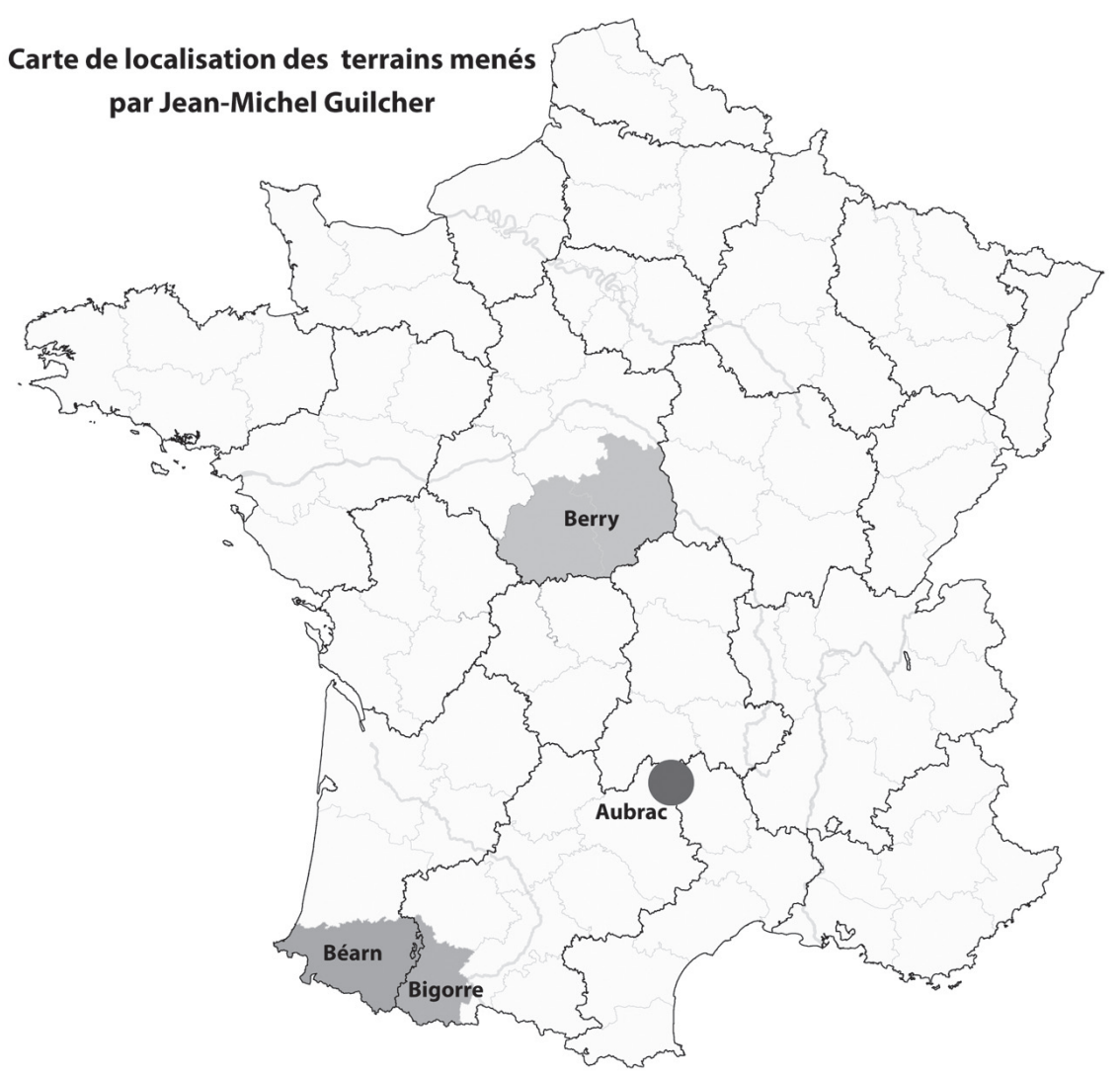

Illustration 2

Les terrains français de Jean-Michel Guilcher en dehors de la Bretagne Carte dessinée par Céline Siguret, UBo, Brest

en Bretagne s'effectue à titre privé. Fort de premiers résultats significatifs, Jean-Michel Guilcher est allé voir l'abbé Falc'hun (1909-1991). Membre de la Société d'ethnographie française, linguiste, ce dernier est une personnalité charnière. En 1939 c'est à lui qu'il a été fait appel pour participer à la mission de folklore musical en Basse-Bretagne, initiée par le Musée des AтP ${ }^{17}$. Selon

recherches sur la danse traditionnelle en France ", Cahiers d'ethnomusicologie [en ligne], 21/2008, p. 258 : ethnomusicologie.revue.org/1297. Consulté le 28 janvier 2015.

17. Marie-Barbara Le Gonidec (dir.), Les Archives de la Mission de folklore musical en BasseBretagne de 1939, Paris, éditions du Стно/Rennes, Dastum, 2009 ; Gilles Goyat : « Un cas atypique : François Falc'hun (1909-1991), le linguiste de la Mission de folklore musical en Basse-Bretagne de 1939 », dans Jean-Pierre Pichette (dir.), L'Apport des prêtres et des religieux au patrimoine des minorités (Actes des journées internationales d'études tenues les 19, 20 et 21 octobre 2011 à l'Université Sainte-Anne et les 17 et 18 novembre 2011 à l'Université de Bretagne Occidentale), Port-Acadie. Revue interdisciplinaire des études acadiennes, $\mathrm{n}^{\circ}$ 24-25-26, automne 2013-printemps 2014-automne 2014, p. 203-212. 
Jean-Michel Guilcher, c'est lui qui l'aurait incité à se lancer dans une thèse ${ }^{18}$.

L'abbé Falc' hun s'occupe également du Bleun Brug («fleur de bruyère »), association catholique qui, dans les années 1950, organise des stages d'été destinés à former jeunes et enseignants à la langue et à la culture populaire bretonnes. Jean-Michel Guilcher est sollicité pour y intervenir, comme il l'est également pour les stages d'été de l'association des instituteurs laïques, Ar Falz (« la faucille »). D'une certaine façon, à défaut de celle de l'institution, il trouve là une forme de reconnaissance. Les comptes rendus sont à chaque fois élogieux quant à la pertinence et à la hauteur de vue du chercheur. Ses conseils, ses critiques sont recherchés et pris en considération. Ainsi s'il intervient lors du stage du Bleun-Brug qui se tient à Quimper du 2 au 6 septembre 1957, pour une conférence remarquée sur « Cultures ethniques et cultures universelles », il est présent quelques jours plus tôt à celui proposé à Glomel (Côtes d'Armor) par l'association Ar Falz qui organise une « Semaine d'enquête ethnographique » (18-24 août). Au cours de sa conférence (26 août) sur « Les recherches en matière de danses populaires », Jean-Michel Guilcher échange avec les participants sur les méthodes d'enquête, prodigue des conseils pratiques « dont on ne manquera pas de se souvenir et qui éviteront de verser dans bien des erreurs malheureusement trop communes dans les

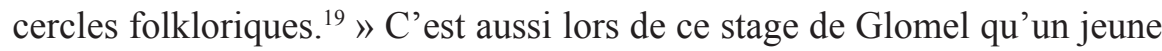
animateur du volet musical, un certain Donatien Laurent, fait la connaissance de celui qui deviendra son maître et dont il suivra les pas à Paris, puis à Brest.

Mais 1957 marque aussi la disparition de Van Gennep, un an après celle de Paul Delarue, deux ans avant celle de Patrice Coirault. Jean-Michel Guilcher aura à cœur de faire connaître l'héritage intellectuel de ces personnalités restées dans l'ombre. Mais il faut ainsi attendre 1994 pour que, sur l'initiative de l'Université de Poitiers, deux journées soient enfin consacrées à l'œuvre de Patrice Coirault, « un savant de premier ordre, autour duquel on fait silence depuis quarante ans » ! dira Jean-Michel Guilcher dans l'intervention qui ouvre ces journées : «L'œuvre de Coirault, ajoute-t-il, demeure peu ou mal connue. À cela deux raisons au moins. L'une tient au tour, trop souvent difficile à suivre, que lui-même a donné à l'expression de sa pensée. L'autre est que nos institutions officielles n'ont mis aucun empressement à prendre en compte la pensée de cet indépendant qui a travaillé loin d'elles et ne leur avait point payé tribut. ${ }^{20} »$

18. François Falc'hun a été le directeur de recherche de Jean-Michel Guilcher avant qu'il ne soit rattaché au musée des ATP (entretien JLG/BMH). Peut-être faut-il voir dans l'intérêt du linguiste pour les travaux de Jean-Michel Guilcher, la communication que ce dernier propose lors du Congrès des sociétés savantes organisé à Rennes en mars 1951 sous le titre : « Une enquête sur les danses bretonnes : but et méthodes ».

$19 \operatorname{Ar~Falz~n}{ }^{\circ}$ 1, janvier-février 1958, p. 24.

20. Jean-Michel Guilcher, "L'Apport de Patrice Coirault à une recherche d'aujourd'hui », 
Nul doute que, par ces réflexions à propos de Coirault, Jean-Michel Guilcher pense également à son propre parcours. La reconnaissance par l'institution sera en effet longue à venir. Il a plus de quarante ans quand il entre au CNRS.

\section{Le versant institutionnel : la carrière d'un enseignant-chercheur}

\section{A. Le chercheur}

Jean-Michel Guilcher a rapporté la manière dont s'est produit son recrutement au CNRS :

Quand [mon] travail [de thèse] a été suffisamment avancé, j'ai présenté ma candidature au CNRS. Je détaillais longuement les questions auxquelles mes recherches s'efforçaient de répondre touchant le fonctionnement de la tradition. Je me rappelle avoir soumis ce texte à Paul Delarue, qui l'approuva chaudement. La commission du CNRS devait en juger autrement. Je n'étais connu de personne, et la danse n'avait pas la réputation d'être un objet d'étude sérieux. L'année suivante, j'ai rendu visite à Valois $[\text { sic }]^{21}$, dont je savais l'intérêt qu'il portait aux sciences naturelles. Je lui ai donné à examiner la collection du Montreur d'images (plantes, oiseaux) dont j'avais eu la responsabilité particulière à l'Atelier du Père Castor. Valois a soutenu ma candidature et, en 1955, je suis entré au CNRS ${ }^{22}$.

Nous avons pris le parti de cette longue citation parce qu'elle nous paraît significative du tournant que prend la carrière de Jean-Michel Guilcher au milieu de ces années 1950 : il est encore un inconnu, dit-il ; son sujet de recherche ne présente aucun intérêt pour les sommités scientifiques chargées de pérenniser la recherche en sciences sociales en France et ce sont finalement des compétences en sciences naturelles qui auraient motivé son recrutement en sciences... humaines!

Pour autant, c'est dans le cadre institutionnel du CNRS qu'il poursuit et mène à bien ses recherches concernant La Tradition populaire de danse en Basse-Bretagne, dont les résultats sont publiés en $1963^{23}$.

Entre-temps, en 1958, il a rejoint le Musée national des arts et traditions populaires : il y est invité par Georges Henri Rivière pour fonder un

Autour de l'œuvre de Patrice Coirault, Actes du colloque organisé par l'Université de Poitiers (Département de musicologie) les 24 et 25 novembre 1994, réunis par Joseph Le Floch, FAMDT, 1997, p. 16.

21. Henri-Victor Vallois (1889-1981). Voir l'article d'Yves Coppens : www.persee.fr/web/revues/home/prescript/article/bmsap_0037-8984_1982_num_9_2_9753.

22. Yves Defrance et Jean-Michel Guilcher, "Jean-Michel Guilcher. Un demi-siècle de recherches sur la danse traditionnelle en France », Cahiers d'ethnomusicologie [en ligne], 21/2008, p. 259 : ethnomusicologie.revue.org/1297. Consulté le 13 septembre 2014. Jusque-là, ses articles sont cosignés avec Hélène Guilcher : après ce n'est plus le cas. Posture institutionnelle oblige ?

23. Jean-Michel Guilcher, La Tradition populaire de danse en Basse-Bretagne, Mouton, ParisLa Haye, 1963 et 1976. L'ouvrage a été réédité en 1995 par les maisons d'éditions Chasse-MaréeArMen à Douarnenez et Coop-Breizh à Spézet, précédé d' « Un regard sur une recherche ancienne » par Jean-Michel Guilcher (p. I-XXXVIII). Il s'agit de la publication d'une thèse d'État menée sous la direction d'André Leroi-Gourhan. 
département de choréologie. Depuis sa création, cette institution muséale est plus qu'un musée puisqu'elle est aussi constituée en centre de recherche, le Centre d'ethnographie française dont les travaux vont progressivement contribuer à définir les concepts et méthodes d'une discipline émergente, alors appelée « ethnographie métropolitaine $»^{24}$. L'ambition de cette nouvelle discipline est de dépasser le projet du folklore désormais considéré comme suranné. Martine Segalen qui fut elle-même directrice du dit musée, nous rappelle opportunément que Jean-Michel Guilcher fut un de ceux qui, « dès les années 1960 [...] inventèrent une nouvelle ethnologie, abandonnant les grandes enquêtes extensives [...] au profit de travaux de terrain intensifs ${ }^{25} \gg$. Dans le cadre de ses nouvelles fonctions, Jean-Michel Guilcher poursuit donc ses recherches sur les danses traditionnelles, menant notamment en Berry, en Béarn, en Bigorre, en Aubrac des terrains dont les résultats sont publiés entre $1965^{26}$ et $1984^{27}$ (ill. 3).

Les recherches qu'il mène en Aubrac participent en réalité d'une démarche pluridisciplinaire qui, à l'instigation du Musée, fait collaborer, à partir de 1963, les ethnologues des ATP avec des sociologues du CNRS. Ce qui, toujours selon Martine Segalen, aurait produit des tensions ayant ellesmêmes connu des prolongements institutionnels ${ }^{28}:$ de fait, le $1^{\text {er }}$ janvier 1968, le sociologue Jean Cuisenier qui avait lui-même participé à l'aventure aubracienne, succède à Georges Henri Rivière à la tête du musée. Le nouveau directeur entreprend alors d'en "redéfinir [et 1]es objectifs et [1] a mission ${ }^{29}$ ». Jean-Michel Guilcher n'y aurait-il plus trouvé son compte ? Toujours est-il qu'il quitte alors le Centre d'ethnologie/d'ethnographie française et qu'il vient à Brest renforcer le Centre de recherche bretonne et celtique où il joue d'emblée un rôle de premier rang puisqu'il en devient le directeur adjoint. Son arrivée a probablement été préparée par le chanoine Falc'hun. Probablement aussi par Yves Le Gallo, directeur-fondateur du CRBC : c'est du moins ce qui parait ressortir des recherches menées par Yvon Tranvouez qui en a esquissé

24. Martine Segalen, Vie d'un musée, op. cit., p. 91. (Cf. le titre de l'ouvrage de Marcel Maget: Ethnographie métropolitaine. Guide d'étude directe des comportements culturels, Paris, Civilisations du Sud, 1953.

25. Id., p. 224.

26. Cf. Jean-Michel Guilcher, «Les Formes anciennes de la danse en Berry », Arts et Traditions populaires, janvier-mars 1965 , p. 3-34.

27. Cf. Jean-Michel Guilcher, La Tradition de danse en Béarn et Pays Basque français, Paris, Maison des sciences de l'homme, 1984, $727 \mathrm{p}$.

28. Cf. Martine Segalen, «L'Enquête de la Rcp Aubrac (1963-1966). Une stratégie intellectuelle, un enjeu institutionnel ", En France rurale. Les enquêtes interdisciplinaires depuis les années 1960, Bernard Paillard, Jean-François Simon et Laurent Le Gall (dir.), Rennes/Brest, Presses universitaires de Rennes/Centre de recherche bretonne et celtique, 2010, p. 263-279.

29. Donatien Laurent, « De la Bretagne au Pays Basque. Un chercheur de renommée internationale », ArMen, n 67, 1995, p. 22-23. 


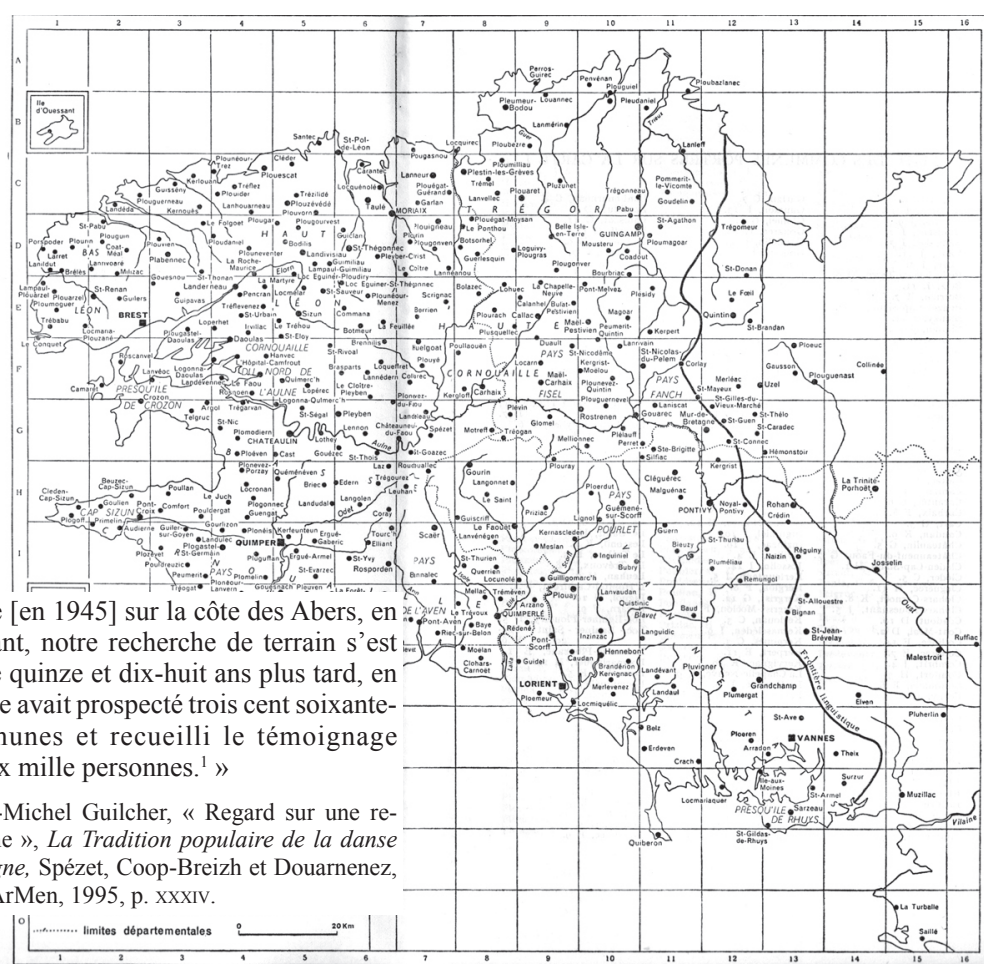

« Commencée [en 1945] sur la côte des Abers, en face d'Ouessant, notre recherche de terrain s'est achevée, entre quinze et dix-huit ans plus tard, en pays gallo. Elle avait prospecté trois cent soixantequinze communes et recueilli le témoignage d'environ deux mille personnes. ${ }^{1}$ »

1. Jean-Michel Guilcher, " Regard sur une recherche ancienne ", La Tradition populaire de la danse en Basse-Bretagne, Spézet, Coop-Breizh et Douarnenez, Chasse-Marée/ArMen, 1995, p. XXXIV.

\section{Illustration 3}

Carte des communes bretonnes étudiées par Jean-Michel Guilcher extraite de La Tradition populaire de la danse en Basse-Bretagne, Paris-La Haye, Mouton, 1963

les premiers résultats lors d'une communication au Jubilé scientifique de Jean-Michel Guilcher, tenu à Brest en octobre 2014 (ill. 4) ${ }^{30}$.

En arrière-plan de ces conflits de disciplines et peut-être aussi de personnes, dont le MNATP fut le théâtre, il est un autre élément qu'il faut sans doute prendre en considération, mais qui ne concerne cette fois que la seule science ethnologique : à la fin des années 1960 en effet, la discipline ellemême en vient à renouveler ses objectifs et ses méthodes. La société française connaît alors de profonds bouleversements produits par la modernisation et l'ethnologie, qui se veut être de son temps, n'entend plus se satisfaire des objets d'étude qui la ramèneraient à un passé, même récent, et qui étaient déjà ceux du folklore : plus que jamais la tradition tient d'un « fumet vieillot ${ }^{31}$ »

30. Cf. Yvon Tranvouez, « Une énigme et un paradoxe : Jean-Michel Guilcher, directeur adjoint du Centre de Recherche Bretonne et Celtique (1969-1979) » qui avance l'idée que l'existence d'une recherche ethnologique au $\mathrm{CRBC}_{\mathrm{RB}}$, incarnée par Jean-Michel Guilcher, pouvait être un argument de taille avancé par Yves Le Gallo, son fondateur, pour que son implantation se fît au final à Brest plutôt qu'à Rennes qui y prétendait également.

31. Martine Segalen, «L'Enquête de la RcP Aubrac », op. cit. : p. 263. 
qui n'intéresse plus guère que des... «passéistes ». Or Jean-Michel Guilcher ne mène-t-il pas précisément sa réflexion sur la notion de tradition?

C'est de cette question en effet qu'il vint à Brest nous entretenir...

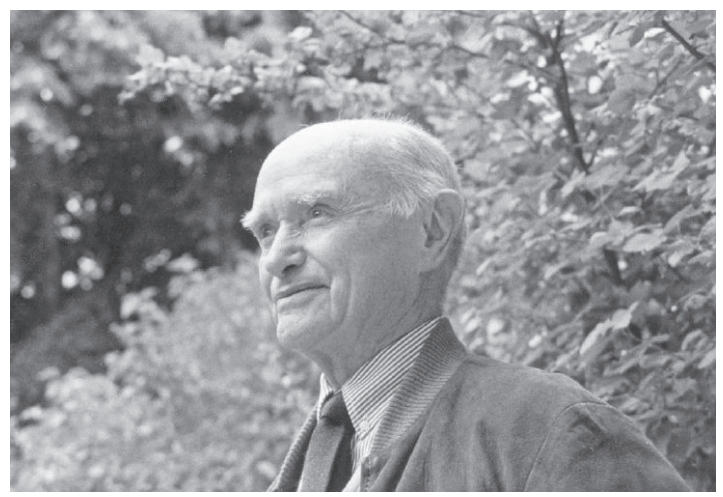

Illustration 4

Jean-Michel Guilcher, le fondateur de l'ethno-choréographie française, a eu 100 ans le 24 septembre 2014

Jubilé scientifique de Jean-Michel Guilcher

18 octobre, Créteil ; 14 et 15 novembre 2014, Brest

Photo : Ph.-E. Raviart

\section{B. L'enseignant}

Les archives en témoignent : dès sa création, Jean-Michel Guilcher est membre du CRBC. À l'évidence, il y trouve, exprimés ou larvés, des projets de recherche qui rejoignent et confortent sa position personnelle. Comme au MNATP, du temps où « l'ethnographie folklorique, prévoyante fait [d'abord] ses conserves, préparant ainsi ses futurs travaux de comparaison et d'interprétation $^{32}$ », l'ambition affichée au CRBC est de se lancer « dans une entreprise de "sauvetage", avant qu'il ne soit trop tard : [...] recueillir [...] les ultimes témoignages concernant la civilisation populaire bretonne d'avant 1914, dans les traits archaïques qui pouvaient encore la caractériser ${ }^{33} »$.

Il est difficile, prétentieux même, de résumer en quelques lignes le projet scientifique de Jean-Michel Guilcher, mais l'on doit s'y risquer pour comprendre ce qui va suivre. Jean-Michel Guilcher entend donc questionner la tradition, son contenu, sa transmission. Après avoir un temps adhéré aux thèses folkloristes qui prétendaient - qui prétendent toujours - que la tradition fonctionne comme la mémoire plus ou moins fidèle d'un passé parfois fort reculé, il en vient à s'interroger sur le bien-fondé d'un tel paradigme. Il ne suffit pas de l'affirmer, pense désormais Jean-Michel Guilcher, il faut

32. Martine Segalen, Vie d'un musée, op. cit., p. 80.

33. Archives du CRBC : Yves Le Gallo, « Projet de recherche présenté par le Centre de Recherche Bretonne et Celtique de la Faculté des Lettres et Sciences humaines de Brest », s.d. [vers 1970]. 
l'argumenter : c'est le début d'une longue et minutieuse enquête scientifique. À cette fin, professe-t-il, il faut chercher à comprendre comment fonctionne la tradition et observer comme se produit sa transmission sur la durée, non pas en limant les variations que cette transmission implique, mais au contraire en les prenant comme objets d'analyse. Cette posture explique la démarche de recherche entreprise : à savoir un inventaire aussi exhaustif que possible de toutes les manifestations de toutes sortes de l'objet transmis, un travail, répète-t-il à l'envi, qui est encore loin d'être achevé, mais il faut aussi et surtout une enquête auprès des derniers dépositaires de la tradition qui peuvent -qui pouvaient - en donner, à titre « d'échantillons » - c'était son mot - une « image [...] imparfaite, sans doute, mais [...] irremplaçable ${ }^{34} »$.

D'une telle ambition affichée résulte aussi l'intérêt porté à l'érudition, pourvoyeuse d'informations, qui se double cependant d'une méfiance à l'égard des érudits : d'un côté donc, mener « une entreprise d'inventaire, de classement et de synthèse des multiples matériaux [...] dispersés et inexploités, dans les archives et les bibliothèques ${ }^{35} »$, mais de l'autre, éviter le risque d'une « claustration dans un ghetto érudit ${ }^{36}$ ».

Il reste que seule l'enquête de terrain peut apporter des éléments pour répondre avec précision à un questionnement renouvelé et il faut donc inciter de jeunes chercheurs à la mener... C'est ainsi, pensons-nous, qu'il faut comprendre l'organisation que Jean-Michel Guilcher fait à la rentrée universitaire 1971, à Brest, d'un séminaire que nous qualifierions donc « d'appel». Il s'en est d'ailleurs lui-même justifié :

En revenant en Basse-Bretagne, je me proposais d'étendre mes anciennes enquêtes à des objets nouveaux (notamment au mobilier traditionnel) et simultanément d'y chercher une réponse à quelques problèmes posés par le fonctionnement culturels dans les anciens milieux de tradition orale. Le souvenir que je gardais de mon premier terrain de recherche m'y faisait espérer des résultats encore substantiels. Les sondages préparatoires auxquels j'ai procédé dans une vingtaine de communes, m'ont fait constater que là comme ailleurs le souvenir des traditions anciennes achevaient de disparaître en même temps que les objets de la culture matérielle, et qu'une recherche personnelle de grande étendue, à échéance lointaine, n'avait plus aucune chance d'atteindre ses buts. Une recherche d'équipe pouvait seule nourrir encore quelque ambition. La tâche la plus urgente était donc de mettre sur pied un enseignement de formation à la recherche en domaine bretonnant ${ }^{37}$.

34. Jean-Michel Guilcher, Danse traditionnelle et anciens milieux ruraux français, op. cit., p. 7.

35. Archives du CRBC, Yves Le Gallo, «Projet de recherche présenté [...] », op. cit.

36. Selon Jean-Michel Guilcher (Archives du CRBC, compte rendu de la réunion du 14 octobre 1969).

37. " Titres et travaux de Jean-Michel Guilcher Maître de recherche CNRS », dactylographié, [s.d.], p. 5-6 (Bibliothèque Yves-le-Gallo). 
Il s'agit d'un enseignement informel, dispensé à titre bénévole, que donne Jean-Michel Guilcher trois années de suite. Cet enseignement s'adresse à un public élargi, d'étudiants sans doute qui pourtant n'y trouvent pas matière à évaluation, mais aussi d'auditeurs extérieurs qui chaque semaine viennent écouter le conférencier et partager avec lui, sur les différents thèmes qu'il aborde, les usages et les représentations dont ils ont connaissance et qui tiennent plus du témoignage direct ou indirect que du résultat d'une investigation personnelle : le temps n'est pas encore venu de lancer une véritable démarche d'enquête dont le CRBC a cependant le projet. Ces séminaires « d'appel » traitent tous d'un thème relatif à la Bretagne : « Le cycle de l'existence individuelle », en 1971-1972, « Le travail paysan [au XIX ${ }^{\mathrm{e}}$ siècle] », en 1972-1973, une " Introduction à l'étude des littératures populaires de tradition orale », en 1973-1974 ${ }^{38}$.

Dans le courant de l'année 1974, l'opportunité se présente de créer à Brest un véritable enseignement d'ethnologie. L'historien Jacques Le Goff est alors président de la $\mathrm{VI}^{\mathrm{e}}$ Section de l'École pratique des hautes études (ÉPHÉ). Il est lié à Jean-Michel Guilcher et à Donatien Laurent par une relation d'estime tout au moins ${ }^{39}$. L'enseignement envisagé se justifie par la politique de décentralisation scientifique alors mise en œuvre : c'est ainsi qu'est créé à l'Université de Brest, en mai 1976, une antenne de l'École des hautes études en sciences sociales (ÉHÉss), le Centre d'ethnologie de la France dont la direction est bien entendu assurée par Jean-Michel Guilcher.

Dès la rentrée universitaire de cette même année 1976, les séminaires reprennent et portent, dans une perspective plus générale, sur « l'étude des anciennes sociétés rurales du domaine français et de leur culture traditionnelle ${ }^{40} »$. Pour sa part, Jean-Michel Guilcher traite personnellement des thèmes suivants : « La tradition (contenu et transmission) », en 1976-1977, « Les méthodes de recherche » et « La littérature populaire de tradition orale », en 1977-1978, « La poésie chantée [selon l'œuvre de Patrice Coirault] », en 1978-1979. Cette même année universitaire il fait aussi le « Bilan du séminaire 1976-1979 : tradition et élaboration folklorique ${ }^{41} »$ : en effet, en décembre 1979, atteint par la limite d'âge, il quitte le CRBC et l'Université de Bretagne occidentale.

Faute de voir renouveler ses financements, le Centre d'ethnologie de la France cesse ses activités dès 1980 quand Donatien Laurent se voit signifier par les instances universitaires la fin de cette expérience de décentralisation

38. Notes personnelles J.-F.S.

39. Jacques Le Goff, «L'Appétit de l'histoire », Essais d'ego-histoire. Maurice Agulhon, Pierre Chaunu, Georges Duby, Raoul Girardet, Jacques Le Goff, Michelle Perrot, René Rémond (Pierre Nora, dir.), Paris, Gallimard, 1987, p. 229.

40. Archives EHÉss, Fonds Le Goff, 19920571/5, communiquées par François Gasnault.

41. Notes personnelles J.-F.S. 
d'un enseignement d'ethnologie à Brest. Elle laissait un goût d'inachevé, mais il faut dire qu'elle avait rencontré des difficultés de fonctionnement, dues en partie à l'éloignement de Brest par rapport à Paris et à l'ÉHÉss : ce dont témoignent les avions de Nicole Belmont régulièrement déroutés sur Nantes ou Lorient ou les polycopiés que Jean-Michel Guilcher devait se charger de présenter et de commenter en l'absence de Marie-Louise Tenèze. En outre le Centre n'avait vu se créer aucun des postes (secrétariat, documentation) prévus et avait dû compter sur l'appui administratif du CRBC.

Mais, malgré la brève existence du Centre d'ethnologie de la France, on ne peut toutefois parler d'échec, tant il est certain que, sans cette première expérience non aboutie, il n'existerait probablement pas aujourd'hui de département d'ethnologie à Brest : ouvert en 1990 à l'Université de Bretagne occidentale, c'est à lui que nous devons tous ces échanges scientifiques établis depuis plusieurs décennies entre Brest, le CRBC et les différents organismes de recherche franco-canadiens, dont Jean-Pierre Pichette a, dans son allocution d'ouverture, rappelé toute la richesse. Ils nous conduisent une nouvelle fois à participer à ce que nous avons convenu d'appeler « Parcours comparés Bretagne/Canada français ", en rapprochant les itinéraires de deux personnalités marquantes dans le passage du folklore à l'ethnologie.

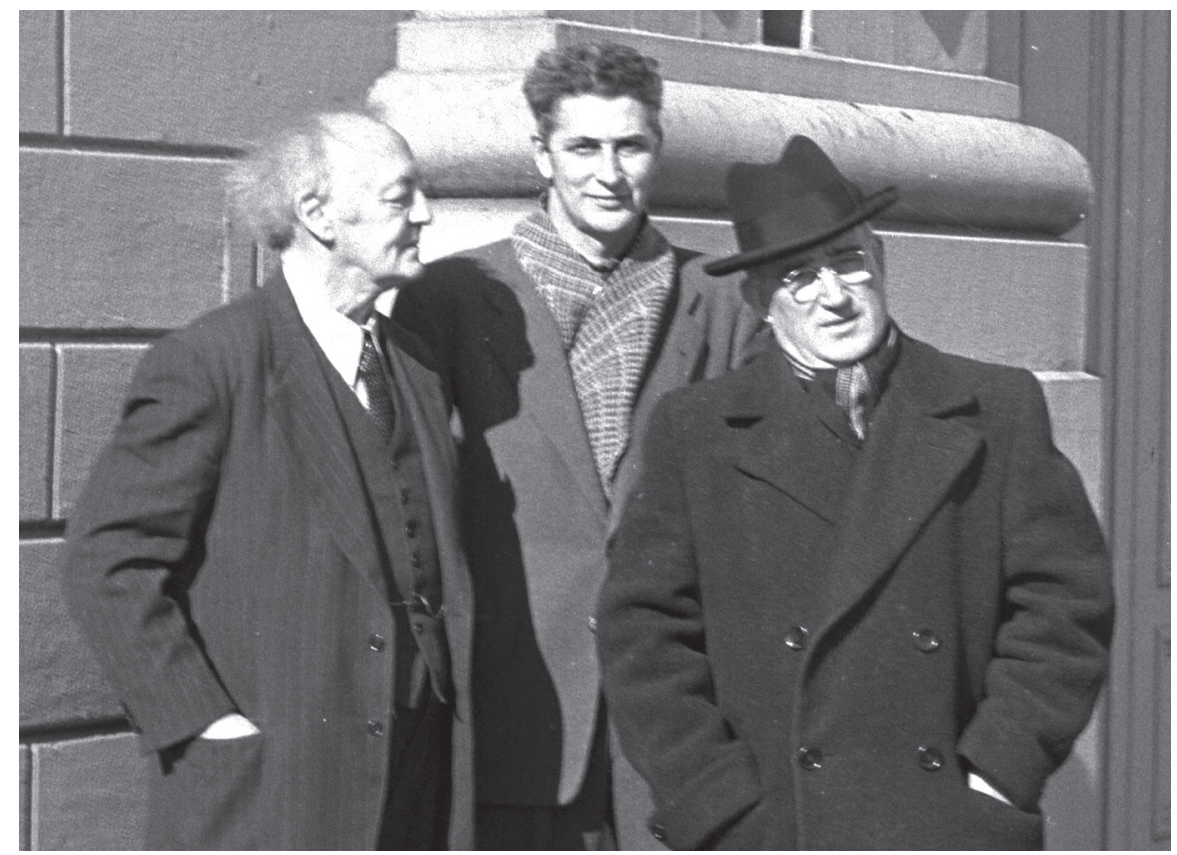

Marius Barbeau, Luc Lacourcière et $\mathrm{M}^{\mathrm{gr}}$ Félix-Antoine Savard à l'Université Laval, Québec, 1956.

Source : Musée canadien de l'histoire nº J13918. 